\title{
Application of Artificial Neural Network in Predicting Flashover Behaviour of Outdoor Insulators under Polluted Conditions
}

\author{
Umer Sajjad \\ Department of Electrical Engineering \\ HITEC University \\ Taxila, Pakistan \\ Jawad Ahmad \\ School of Computing \\ Edinburgh Napier University \\ Edinburgh, UK
}

\author{
Arshad \\ Institute for Energy and Environment \\ University of Strathclyde \\ Glasgow, UK \\ Sultan Shoaib \\ Faculty of Art, Science and Technology \\ Wrexham Glyndwr University \\ Wrexham, UK
}

\begin{abstract}
Safe and reliable delivery of power through transmission lines mainly depends on the quality condition of the high voltage insulators. In the last few decades, demand in polymeric insulator has been dramatically increased due to their advanced performance in comparison to ceramic and glass insulators. This paper discusses the application of Artificial Neural Network (ANN) to predict the flashover parameters of polymeric insulators under the impact of weather and environment conditions. The training data for ANN were obtained from experimental tests executed in the climate chamber with the implementation of high voltage stress. The parameters predicted in this paper are arc-inception voltage, flashover voltage and surface resistance. A promising application of the ANN model proposed in this paper is the effective prediction of the flashover parameters of polymeric insulators affecting by extreme temperature, humidity and pollution level. These results will also enhance our understanding of the flashover process in outdoor polymeric insulators.
\end{abstract}

Keywords-neural network, flashover behaviour, high voltage, insulators

\section{INTRODUCTION}

Outdoor insulators are major parts of transmission and distribution system. These insulators are exposed to many stresses like mechanical, environmental and electrical during service conditions. Flashover on outdoor high voltage insulators is considered to be the primary problem affecting the reliability of transmission lines' operation. Flashover on outdoor insulators may be due to many reasons. However, air pollution is considered one of the major source of triggering a flashover on insulators [1]. Different types of material are used for outdoor insulation such as ceramic, glass and polymer. Due to the hydrophobic nature of polymeric material, it is considered to have better flashover performance under polluted conditions [2]. Furthermore, comparing to other insulators, polymeric insulators demonstrate significant improvement in terms of cost, weight, installation and maintenance. However, due to organic nature, polymeric insulators are susceptible to tracking and treeing [3].

The benefits of polymeric insulators above ceramic and glass insulators are related to their superior property of hydrophobicity (capability of a material to resist the construction of continuous water layers on its surface). Under wet conditions, water drops deposit on the surface of hydrophobic insulator [4]. These high electric fields at the junction result into water drops deformation in the direction of applied electric field stress. Under certain conditions, when the electric field stress is very high, it may result in the initiation of electric arcs. Although these partial arcs are of low energy, it may result in surface degradation of polymeric insulators if exist for a longer duration. It may also result in non-uniform current density, Joule heating and dry band formation along the insulator surface. The voltage drop along the dry bands increases further and may result in flashover.

Pollution flashover in outdoor insulators is dependent on many parameters such as ambient temperature, relative humidity, geographical location, insulator design and material [5]. The chemical constituents of pollution deposited on the insulator surface also affect flashover performance. [6] concluded that the flashover voltage of outdoor insulators depends on the soluble substance, their type and chemical composition. Daochun, et al. [7] reported that Non-Soluble Salt Deposit Density (NSDD) also have an impact on flashover characteristics and in the slurry the size of particles in nonsoluble material is the primary reason affecting flashover. Another important factor is humidity which affects the features of outdoor insulators. It has been reported in [8] that relative humidity is directly proportional to the breakdown strength of air gaps and corona discharges. Similarly, for ice-covered 
insulators, inception voltage increases by $3 \%$ when humidity was altered from low to medium level [9]. However, it was reported in [10] that in the presence of insulation surface, the flashover voltage decreases with an increase in humidity. It was also concluded that a decrease in flashover voltage is mainly because of the increase in surface conductivity. The behaviour of outdoor high voltage insulators under polluted environments has been investigated by many researchers in laboratory.

As laboratory experiments need a sensitive hardware and software. This leads scientists and engineers to investigate alternative methods of forecasting the flashover performance of overhead line insulators. Most of the previous work is based on developing mathematical models for polluted outdoor insulators. However, mathematical models are mostly based on certain assumptions and may not be the accurate representation of field conditions.

Furthermore, the existing intelligent systems do not consider surface resistance which is vital for calculating the performance of outdoor insulators. The previous statistical models to predict surface resistance were developed by Gorur et al. [11]. This paper proposed an ANN algorithm which predicts surface resistance, arc-inception voltage and flashover voltage at different values of ESDD, NSDD, temperature and humidity.

\section{EXPERIMENTAL SETUP}

Tests were conducted under controlled laboratory conditions on the artificially polluted rectangular silicone rubber sheet. The properties of samples used for the tests are given in Table I.

TABLE I. PARAMETERS OF HTV SiliCONE RUBBER

\begin{tabular}{|l|l|}
\hline \multicolumn{1}{|c|}{ Name } & \multicolumn{1}{c|}{ Values } \\
\hline Silicone rubber & GP70THT \\
\hline Temp range & $60-300^{\circ} \mathrm{C}$ \\
\hline Thermal conductivity & $0.24 \mathrm{Wm}^{-1} \mathrm{~K}^{-1}$ \\
\hline Dielectric constant & 2.9 \\
\hline Dissipation factor & 0.0003 \\
\hline Dimensions & $10 \times 4 \times 0.6 \mathrm{~cm}^{3}$ \\
\hline
\end{tabular}

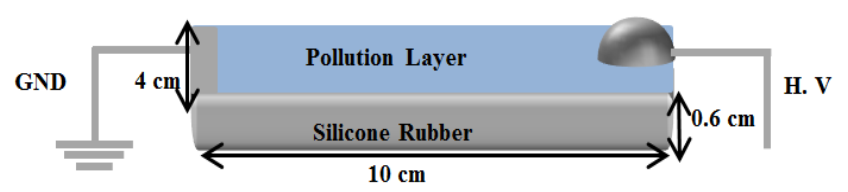

Fig. 1. Test sample.

Artificial pollution was applied on samples by modified solid layer technique (IEC 60507). To minimize the effect of surface hydrophobicity; dry kaolin is used as pre-conditioner. Wettability class is used to check the hydrophilic behaviour of the samples. Whereas Triton X-100 also used as a wetting agent in pollution suspension. Whereas the pollution suspension consists of $1 \mathrm{~g}$ of Triton $\mathrm{X}-100,40 \mathrm{~g}$ of kaolin and $\mathrm{NaCl}$ as per requirement; depending upon the suspension conductivity. To prepare the samples of different NSDD and ESDD level, we varied the amount of kaolin and kept the amount of $\mathrm{NaCl}$ constant. The test sample with uniform pollution layer is shown in Fig. 1.

For experiments, the simplified test setup is shown in Fig. 2. The samples were placed inside the climate chamber horizontally on an acrylic glass stand. The samples were stressed by a power frequency 0 to $100 \mathrm{kV}$ test transformer. The maximum over-voltage and test current were set to $50 \mathrm{kV}$ and 1 A respectively. A data acquisition system has been built using LabVIEW software. The system collects and stores the values of current and voltage data. The details of sample preparation and test setup can be found in [3].

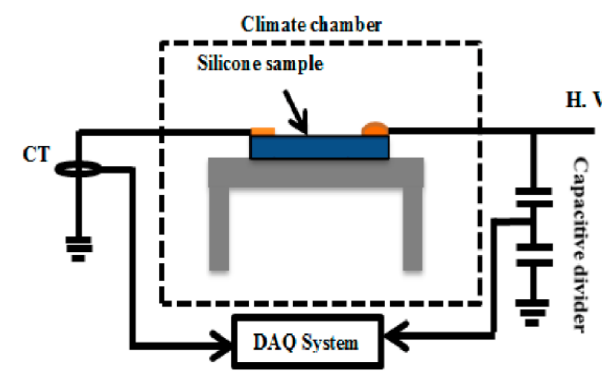

Fig. 2. HV test setup.

\section{ARtificial NeURAL NetWork}

ANN approach was successfully implemented in a large variety of control applications. Recently, ANN-based algorithms have been introduced to estimate the performance of outdoor insulations, including flashover voltage and surface condition. ANN has the ability to use experimental data and model a problem with considerable accuracy. Based on the learning set of data, the model can predict the output for a given set of input variables. The ANN model contains an input layer, hidden layer and output layer. The first layer named as input layer includes input neurons, while the output layer comprises of output neurons. There can be one or more hidden layer in a system. The neurons of hidden layers are connected with each other as well as input and output layer. This paper discusses the model where the number of neurons presented in the hidden layer is 10 . The activation function used in this model is a Hyperbolic sigmoid function.

\section{A. Back Propagation}

Back propagation is a technique used in the neural network to train neurons. In back propagation the system does not need a transfer function but have the values of outputs for particular inputs [12]. On the basis of these values, the neural network calculates the weight of neuron present in hidden layers and check the precision of results [13]. These results are then compared with the data obtained from experiments. If the accuracy is achieved as per set requirement, then the training process would stop. If not, then this process continues to recalculate the weights of the hidden layer until the required accuracy is achieved. Quasi-Newton back propagation is one of the modified Newton's methods that has been used in this paper. This method is used for fast optimization rather than conjugate gradient method [14]. Its general form is given by equation (1).

$$
x_{k+1}=x_{k}-A_{k}^{-1} g_{k}
$$


where $k$ is the number of iterations, $A_{k}^{-1}$ is the Hessian Matrix, and $g_{k}$ is the gradient. The Hessian matrix consists of second derivatives that are the performance index of respective weights of neurons. The conditions of termination of training are shown in Table II.

In this paper, we develop a neural network with four inputs (temperature, humidity, NSDD, and ESDD) and three outputs (surface resistance, inception and flashover voltages). The total number of hidden layers is two. The network is shown in Fig. 3. A total of 101 samples were used in the model. Out of the total data point, $75 \%$ were used for training, $13 \%$ for cross-validation and $12 \%$ for testing. It is important to note here that due to limited experimental data for temperature and relative humidity, the cross-validation was done at $8 \%$ in both cases.

TABLE II. CONDITIONS OF TERMINATION OF QUASI-NEWTON BACK PROPAGATION

\begin{tabular}{|l|l|}
\hline \multicolumn{1}{|c|}{ Criterion } & \multicolumn{1}{c|}{ Conditions } \\
\hline \multirow{4}{*}{ Training process stops if } & Max iteration reached \\
\cline { 2 - 2 } & Max time reached \\
\cline { 2 - 2 } & Desired performance reached \\
\cline { 2 - 2 } & Gradient less than min value. \\
\cline { 2 - 2 } & Validation fails more than defined times \\
\hline
\end{tabular}

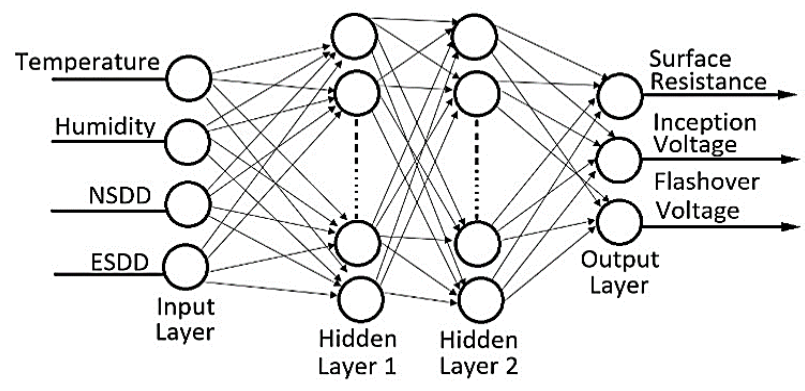

Fig. 3. Test sample.

\section{RESUlTS AND DiscUSSIONS}

This section presents the experimental and predicted results of flashover parameters under different environmental conditions. The effect of ESDD, NSDD, temperature and relative humidity on all three output is described below. The ANN model was run for 1000 iterations, and the minimum error was obtained at 143 iterations. A fitting curve between experimental and predicted results showed 99\% accuracy.

\section{A. Effect of ESDD}

ESDD is one of the most significant parameters affecting the flashover behaviour of outdoor insulators [3], [15], [16]. Pollution dumped on insulator's surface may consist of soluble and non-soluble constituents. In this section, the effect of soluble contaminants is presented. The value of ESDD was varied for fixed values of temperature, relative humidity and NSDD, as shown in Table III.

It displays the experimental and predicted data of surface resistance for various values of ESDD. It can be seen that as the value of ESDD was increased, surface resistance decreases. This is mostly due to the rise in conductivity of pollution deposit. Furthermore, the experimental and predicted results are in good arrangement.

TABLE III. CONSTANT PARAMETERS DURING ESDD TEST

\begin{tabular}{|l|l|}
\hline \multicolumn{1}{|c|}{ Name } & \multicolumn{1}{c|}{ Values } \\
\hline Temperature & $10^{\circ} \mathrm{C}$ \\
\hline Humidity & $70 \%$ \\
\hline ESDD & $0.5 \mathrm{mg} / \mathrm{cm}^{2}$ \\
\hline
\end{tabular}

Arc-inception voltage is the voltage at which the first arc appear and visible on the insulator surface. Measuring and predicting arc-inception voltage help in determining the surface condition of polymeric insulators. In this paper, arc inception voltage was measured for different values of ESDD. The experimental results are presented in Fig. 4 and applied for training ANN model. As reported in the previous section, that increase in ESDD will lead to a decrease in surface resistance.

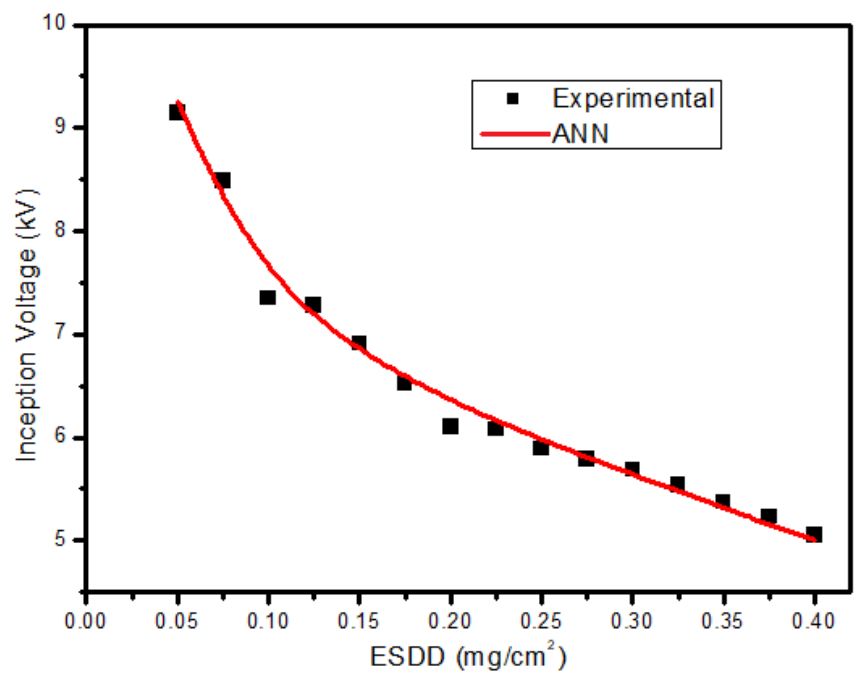

Fig. 4. Experimental and predicted values of inception voltage at different values of ESDD.

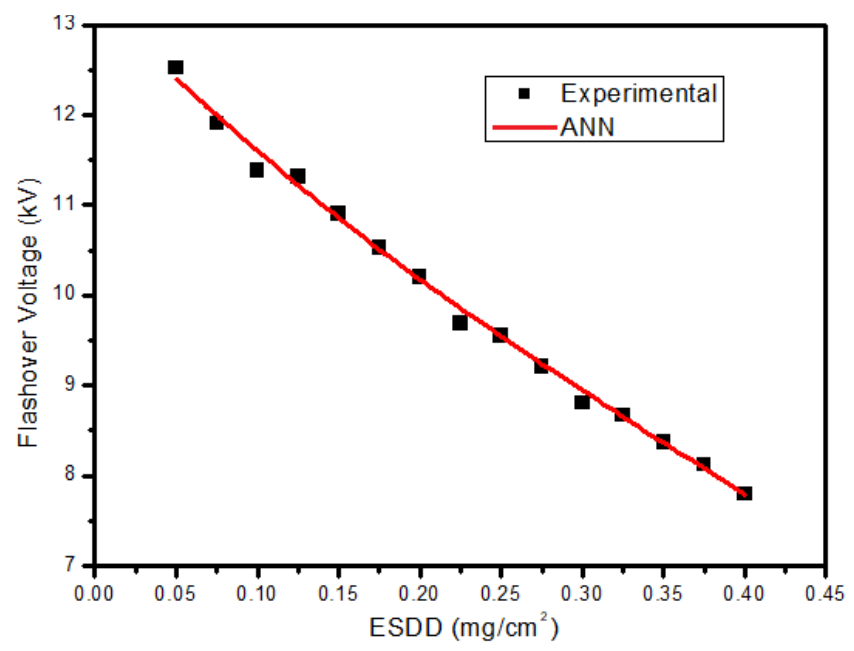


Fig. 5. Experimental and predicted values of flashover voltage at different values of ESDD.

Flashover voltage is one of the significant constraints in characterizing the performance of open-air high voltage insulators. The relationship between flashover voltage and ESDD have been reported in various works of literature. In this paper, flashover voltage was measured experimentally at different values of ESDD and the results were compared with predicted values of flashover obtained from ANN model. The results obtained are reported in Fig. 5 which shows a close arrangement among experimental and predicted results.

\section{B. Effect of NSDD}

As discussed earlier, the pollution deposited on the insulator surface consists of soluble and non-soluble constituents. Although many researchers have investigated the effect of soluble contaminants on flashover voltages of insulators, very little literature exists on the impact of non-soluble contaminants. However, during the experiment, it was found out that nonsoluble impurities also affect the flashover process of polymeric insulators. This effect is dominant in hydrophobic insulators as compared to hydrophilic insulators because the presence of nonsoluble contaminants can disrupt the hydrophobicity recovery process of hydrophobic insulators. In this paper, experimental and predicted results of surface resistance, arc-inception voltage and flashover voltage have been reported under different values of NSDD. Temperature, relative humidity and ESDD were kept constant during this case, as reported in Table IV.

TABLE IV. CONSTANT PARAMETERS DURING NSDD TEST

\begin{tabular}{|l|l|}
\hline \multicolumn{1}{|c|}{ Name } & \multicolumn{1}{c|}{ Valus } \\
\hline Temperature & $10^{\circ} \mathrm{C}$ \\
\hline Humidity & $70 \%$ \\
\hline NSDD & $0.10 \mathrm{mg} / \mathrm{cm}^{2}$ \\
\hline
\end{tabular}

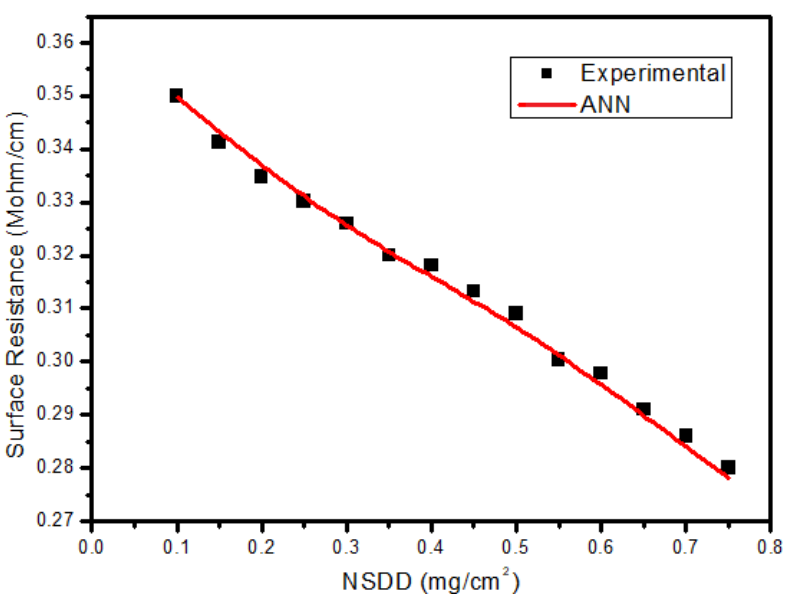

Fig. 6. Experimental and predicted values of SR at different values of NSDD.

Fig. 6 displays the experimental and predicted results of surface resistance at different values of NSDD. It is observed from Fig. 6 that as NSDD increases, surface resistance decreases. This is mainly due to the decrease in surface hydrophobicity of silicone rubber insulator and the development of even the pollution layer, which assists the flow of leakage current.

Fig. 8 shows predicted and experimental results of flashover voltage on various values of NSDD. With the increase in NSDD, a thick layer of pollution form on the surface of the insulator that facilitates the flow of current and decreases flashover voltage. Although the experimental and predicted results are in close arrangement, at higher values of NSDD, the absolute error increases which might be the change in test conditions or the error in the application of pollution layer on the sample.

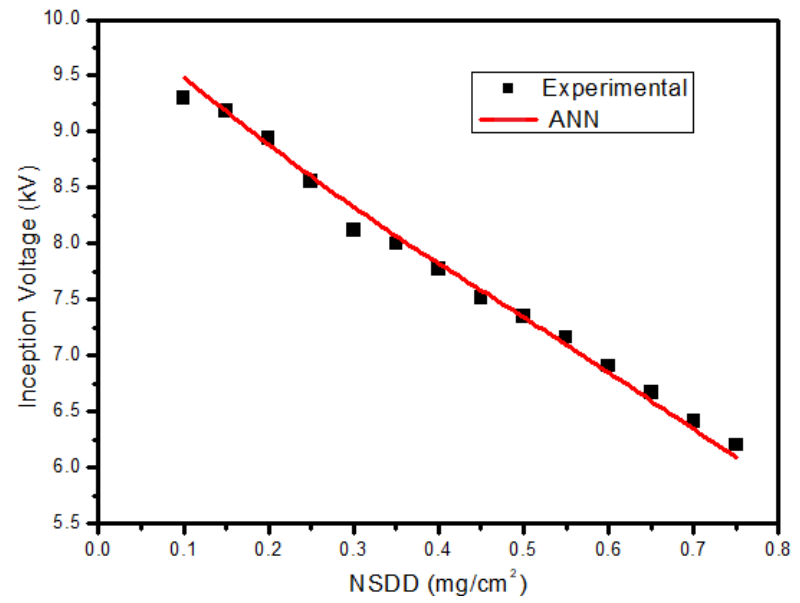

Fig. 7. Experimental and predicted values of inception voltage at different values of NSDD

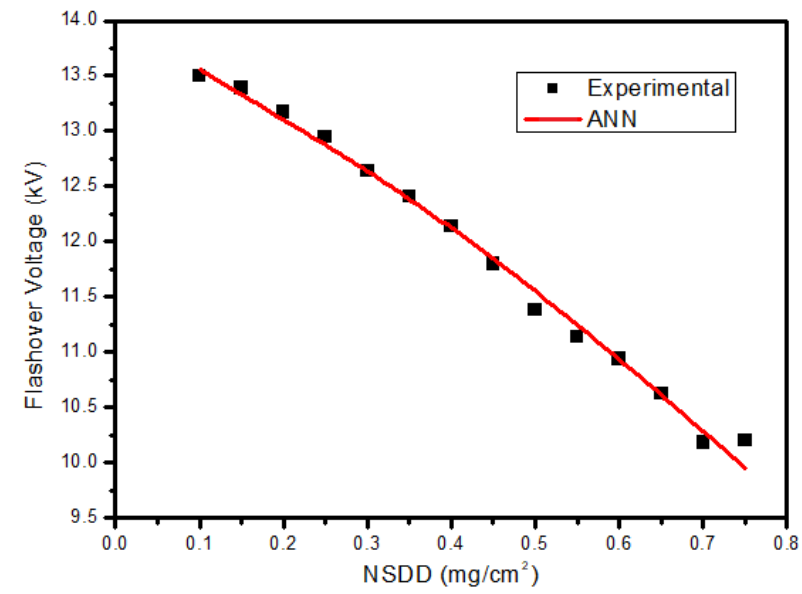

Fig. 8. Experimental and predicted values of flashover voltage at different values of NSDD.

\section{Effect of Relative Humidity}

The relative humidity is another environmental condition which can affect the performance of outdoor insulators. With the increase in relative humidity, the moisture content in the air nearby the insulators will increase. This increase in moisture content may increase surface wetting and increase conductivity. Although the effect of humidity on air gap breakdown has been reported previously in the literature, there is little work done to examine the effect of humidity on flashover performance. In this section, experimental and predicted values of surface resistance, inception voltage and flashover voltage have been presented for different values of relative humidity. Other paraments such as 
ambient temperature, NSDD, ESDD remained constant during the experiment and as reported in Table $\mathrm{V}$.

Table V. Constant Parameters during Relative Humidity Test

\begin{tabular}{|l|l|}
\hline \multicolumn{1}{|c|}{ Name } & \multicolumn{1}{c|}{ Values } \\
\hline Temperature & $10^{\circ} \mathrm{C}$ \\
\hline ESDD & $0.20 \mathrm{mg} / \mathrm{cm}^{2}$ \\
\hline NSDD & $0.75 \mathrm{mg} / \mathrm{cm}^{2}$ \\
\hline
\end{tabular}

Fig. 9 represents the experimental and predicted data of surface resistance at various values of relative humidity. The different values of relative humidity have been applied to conduct the experiments. The results reported in this paper are the mean values of multiple tests. Experimental data was used to train the ANN algorithm. As the relative humidity increases, surface resistance decreases which might be due to the increase in surface wetting and subsequently surface conductivity.

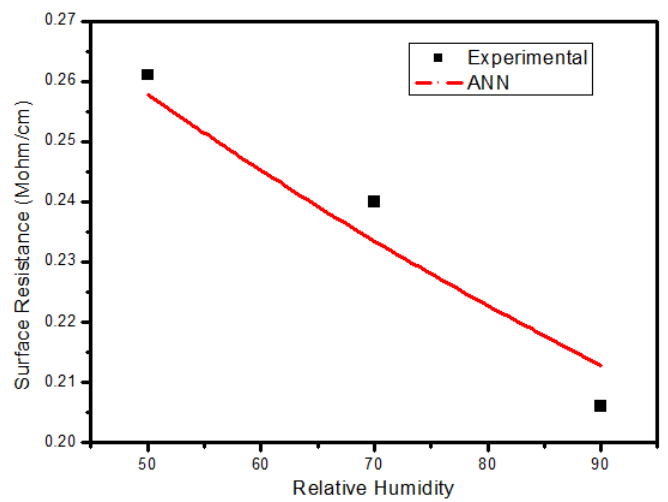

Fig. 9. Experimental and predicted values of SR at different values of relative humidity.

Fig. 10 shows values of arc inception voltage at three different levels of relative humidity obtained from the experiment and predicted. Similar to surface resistance, arc inception voltage is reduced at the increase of relative humidity.

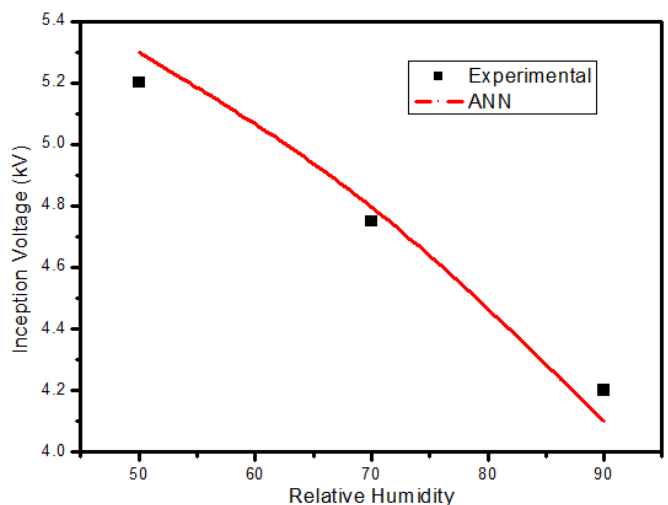

Fig. 10. Experimental and predicted values of inception voltage at different values of temperature.

The impact of relative humidity over flashover voltage has been reported in Fig. 11. There is a sudden decrease in flashover voltage as the level of humidity is varied from the lower value to medium and from medium value to higher. This is mainly due to the decrease in surface resistance which reduces flashover voltage. The predicted results and experimental results are in close agreement.

The training data set used to train the ANN algorithm consists of 77 data samples. In this section, the absolute error was calculated between the experimental and predicted results at each data sample. The absolute error for surface resistance, arc-inception voltage and flashover voltage is given in Fig. 12, Fig. 13 and Fig. 14. By close observation of these plots, it can be revealed that the absolute error is varying for each sample. This is mainly due to the change in experimental conditions. As the pollution was manually applied to the sample and the pollution layer may not uniform in each case. Furthermore, it is difficult to control temperature and humidity within the climate chamber during the test. These variations in test conditions lead to a change in experimental and predicted results. Additionally, there may be an error in the prediction algorithm, which lead to absolute error.

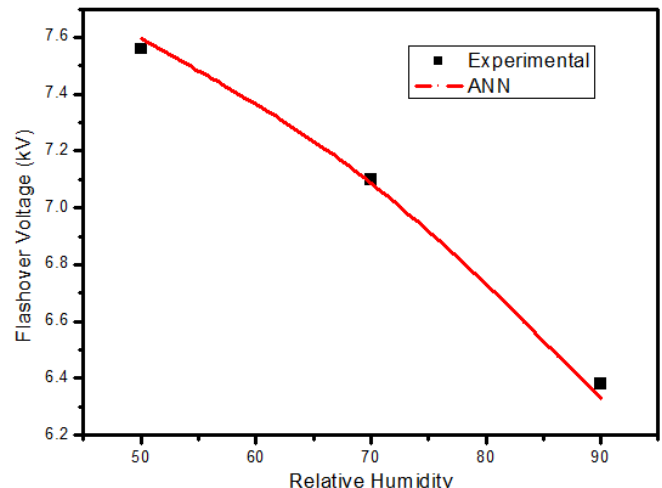

Fig. 11. Experimental and predicted values of flashover voltage at different values of temperature.

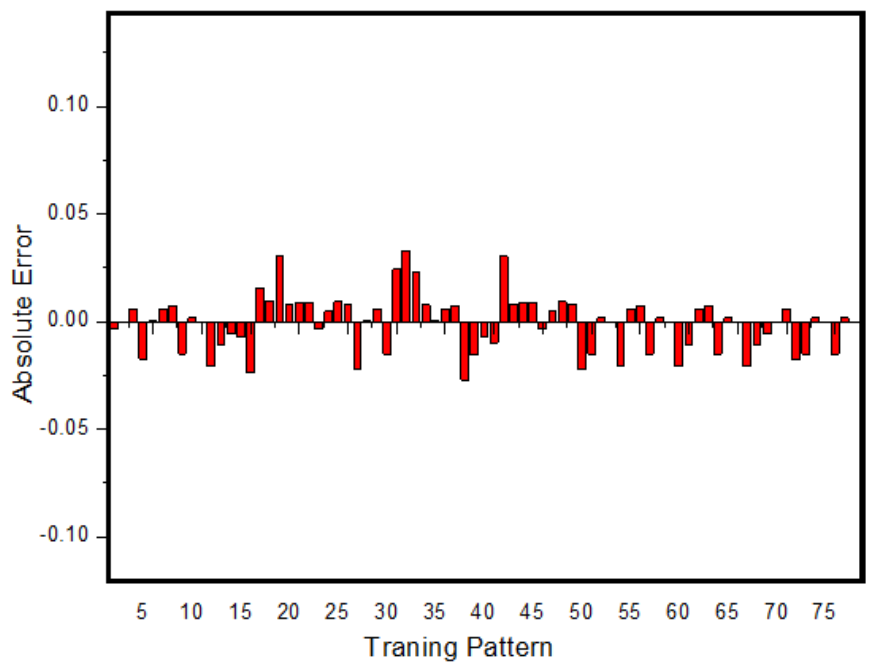

Fig. 12. Absolute error values of surface resistance for all data used in training.

There is an alternative way to check and validate accuracy. According to this alternative approach, we correlate the real and output values of ANN called estimated values. The maximum achievable correlation is 1 , which means $100 \%$ accuracy. The correlation between actual and predicted results is plotted in Fig. 
15. It is clear from the correlation results that the ANN algorithm has an accuracy near ton $99.9 \%$.

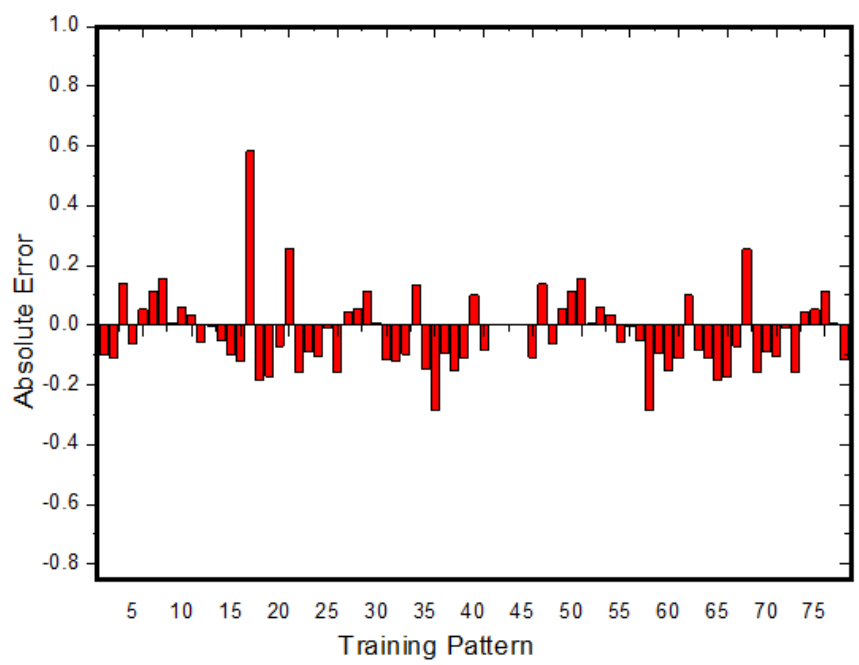

Fig. 13. Absolute error values of inception voltage for all data used in training.

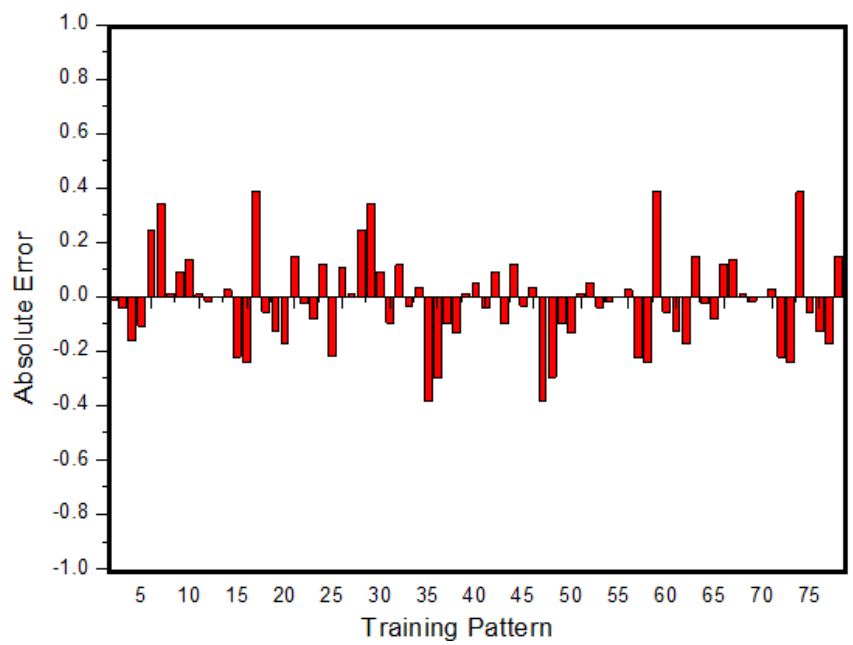

Fig. 14. Absolute error values of flashover voltage for all data used in training.

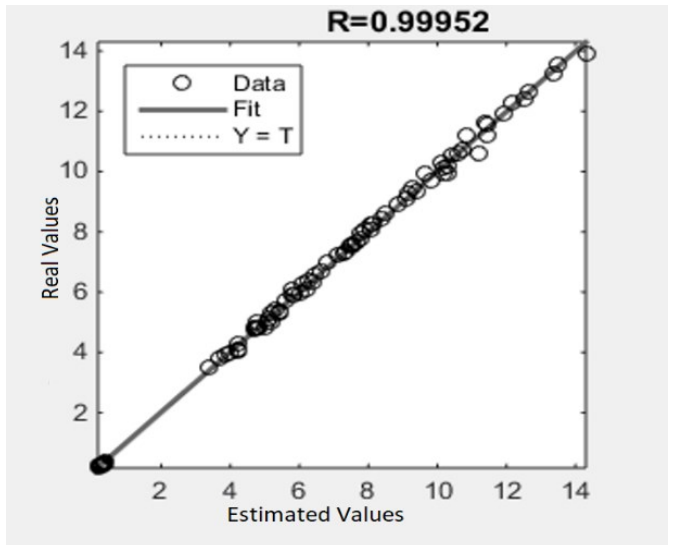

Fig. 15. Correlation between real and estimated values used for training.

\section{CONCLUSION}

This paper introduces a novel ANN algorithm applied to predict flashover performance of outdoor high voltage insulation based on silicone rubber material. Training data was obtained by performing experiments in a high voltage laboratory where the weather and environment conditions have been simulated. The experiments provided the values of surface resistance, arcinception voltage and flashover voltage for various polluted and environment conditions. The paper demonstrates the ability of the proposed ANN algorithm to predict these parameters with considerable accuracy. A promising application of the proposed model is the forecast of the flashover behaviour of outdoor high voltage insulators affecting changing weather and environment circumstances. It will reduce human effort and cost of experimentation at different environmental conditions. These results can be made better by using other neural network techniques and real insulators for experimentation.

\section{REFERENCES}

[1] G.G. Karady, M. Shah, and R.L. Brown, "Flashover mechanism of silicone rubber insulators used for outdoor insulation-I," IEEE Trans. On Power Delivery, vol. 10, no. 4, pp. 1965-1971, 1995.

[2] J. Mackevich, and M. Shah, "Polymer outdoor insulating materials. Part I: Comparison of porcelain and polymer electrical insulation," IEEE Electr. Insul. Mag., vol. 13, no. 3, pp. 5-12, 1997.

[3] N.C. Mavrikakis, P.N. Mikropoulos, and K. Siderakis, "Evaluation of field-ageing effects on insulating materials of composite suspension insulators," IEEE Trans. Dielectr. Electr. Insul., vol. 24, no. 1, pp. 490498, 2017.

[4] F. Aouabed, A. Bayadi, A.E. Rahmani, and R. Boudissa, "Finite element modelling of electric field and voltage distribution on a silicone insulating surface covered with water droplets," IEEE Trans. Dielectr. Electr. Insul., vol. 25, no. 2, pp. 413-420, 2018.

[5] Y.-K. Zhu et al., "Experimental analysis of the artificial contamination flashover characteristics of long insulator strings in $500 \mathrm{kV}$ transmission lines," Advances in Engineering Research, vol. 117, pp. 65-75, 2016.

[6] D. Zhang, Z. Zhang, X. Jiang, Z. Yang, J. Zhao, and Y. Li, "Study on insulator flashover voltage gradient correction considering soluble pollution constituents," Energies, vol. 9, no. 11, Art. no. 954, 2016

[7] D. Huang, W. Lu, Y. Deng, J. Ruan, and Z. Xiong, " $\mathrm{CaSO}_{4}$ content in contamination influence on ac pollution flashover characteristics of XWP 2-160 porcelain insulator string," in Proc. IEEE Int. Conf. on High Voltage Engineering and Application, Athens, Greece, 10-13 Sept. 2018, pp. $1-4$.

[8] E. Kuffel, "Electron attachment coefficients in oxygen, dry air, humid air and water vapour," Proc. Phys. Soc., vol. 74, no. 3, Art. no. 297, 1959.

[9] S. Farokhi, A. Nekahi, and M. Farzaneh, "Mechanisms and processes of arc propagation over an ice-covered surface," IEEE Trans. Dielectr. Electr. Insul., vol. 21, no. 6, pp. 2634-2641, 2014.

[10] J.C. Zheng, Z. Wang, and Y.W. Liu, 'Influence of humidity on flashover in air in the presence of dielectric surfaces," in Proc. IEEE Region 10 Int. Conf. on Computers, Communications and Automation, Beijing, China, 19-21 Oct. 1993, vol. 5, pp. 443-449.

[11] E.D. Karnin, "A simple procedure for pruning back-propagation trained neural networks," IEEE Trans. Neural Netw., vol. 1, no. 2, pp. 239-242, 1990.

[12] M. Cilimkovic, Neural Networks and Back Propagation Algorithm, Inst. Technol. Blanchardstown, Dublin, Irelend, 2015.

[13] J.E. Dennis Jr, and R.B. Schnabel, Numerical Methods for Unconstrained Optimization and Nonlinear Equations, Philadelphia: Siam, 1996.

[14] A. Nekahi, S.G. McMeekin, and M. Farzaneh, "Measurement of surface resistance of silicone rubber sheets under polluted and dry band conditions," Electr. Eng., vol. 100, no. 3, pp. 1729-1738, 2018.

[15] A. Nekahi, S.G. McMeekin, and M. Farzaneh, "Investigating flashover behaviour of silicone rubber insulators under contaminated conditions," in Proc. IEEE Conf. on Electrical Insulation and Dielectric Phenomena, Ann Arbor, MI, USA, 18-21 Oct. 2015, pp. 856-859. 
